\title{
PROPOSTA DE ALTERNATIVAS MENOS TÓXICAS PARA INGREDIENTES ATIVOS DE AGROTÓXICOS NO MERCADO BRASILEIRO
}

\author{
ALLAN VALLE TOLEDO DA SILVEIRA* \\ NELSON ROBERTO ANTONIOSI FILHO**
}

\begin{abstract}
Realizou-se levantamento dos ingredientes ativos (IA) registrados no Brasil, sugerindo alternativas para substituição daqueles com maior toxicidade/impacto ambiental nas culturas de arroz, cana-de-açúcar, café, citrus, milho, feijão, soja e pastagens. As substituições foram propostas para 2,4-D, azociclotina, carbofurano, endossulfan, fenamifós e terbufós. Acredita-se que este estudo possa servir como referência no sentido de auxiliar a formulação de políticas que reduzam os riscos ambientais inerentes ao uso desses insumos.
\end{abstract}

* Doutor em Ciências Ambientais, Laboratório de Meio Ambiente e Recursos Hídricos, Universidade Federal de Goiás (UFG), GO (e-mail: allanvts@ig.com.br).

** Pós-Doutor em Química Analítica, Laboratório de Métodos de Extração e Separação, UFG, GO (e-mail: nelson@quimica.ufg.br). 


\section{INTRODUÇÃO}

A partir do século $\mathrm{XX}$, centenas de ingredientes ativos e milhares de formulações foram criadas e comercializadas para controle de pragas e vetores de doenças na agricultura em todo $o$ mundo. Apesar do benefício decorrente da utilização desses produtos para o aumento da produtividade agrícola e a redução das incidências de pragas, o problema de intoxicações por agrotóxicos tornouse preocupante, sobretudo porque ocorrem pela ingestão gradual desses produtos que contaminam a água, o solo e os alimentos (RIZZATO et al., 2004). Além disso, existem problemas relativos a doenças e mortes humanas ocasionadas pela exposição ocupacional, acidental ou proposital a esses produtos (GARCIA, 1998).

Agrotóxicos são responsáveis por inúmeros efeitos adversos na saúde humana, maior que qualquer outro tipo de intoxicação aguda. Muitos estudos descrevem as associações entre agrotóxicos e vários tipos de doenças degenerativas e diferentes tipos de câncer (DANIELS, OLSHAN e SAVITZ, 1997; KHUDER e MUTGI, 1997; ZAHM e WARD, 1998; ENGEl et al., 2001; GAUTHIER et al., 2001). Seus efeitos nos sistemas imunológico, hematopoiético, nervoso, endócrino e reprodutivo são relatados há muito tempo (OJAJARVI et al., 2000; FIGÁ-TALAMANCA e PETRELLI, 2001; MOURAD, 2005), bem como suas propriedades mutagênicas (COSTA et al., 2007; ERGENE et al., 2007; MUNIZ et al., 2008)

A contaminação por agrotóxicos representa uma das ameaças mais significantes para os ecossistemas (DABROWSKI et al., 2002) e desde o ano de 1940, logo após a descoberta por Müller das propriedades inseticidas do DDT em 1937, estudos indicavam os efeitos danosos desse composto na vida selvagem (RATTNER, 2009). Nos anos de 1950, quando vários outros inseticidas tornaram-se comuns (tais como aldrin, dieldrin, clordano e heptacloro), a mortalidade da vida selvagem foi notada logo em seguida ao uso de agrotóxicos (RATTNER, 2009).

Após a publicação do livro Primavera silenciosa (CARSON, 1962), no início da década de 60, que alertava sobre a presença de compostos orgânicos sintéticos no ambiente e os efeitos devastadores do DDT sobre a vida selvagem, a sociedade começou a pressionar seus governantes no sentido de ampliar o controle sobre as fontes poluidoras.

No Brasil, o conceito de potencial periculosidade ambiental (PPA) dos agrotóxicos foi normatizado em 1996 e definida sua aplicação. O Ministério do Meio Ambiente detinha a competência para essa avaliação, baseada na Instrução Normativa 84/1996 do IBAMA (BRASIL, 1996) que classificou a periculosidade ambiental dos agrotóxicos em Classe I - produto altamente perigoso; Classe II - produto muito perigoso; Classe III - produto perigoso e Classe IV - produto pouco perigoso. Os parâmetros utilizados para tal classificação basearam-se no transporte, persistência, bioacumulação, toxicidade a organismos e potencial mutagênico dos produtos.

Os agrotóxicos foram classificados, ainda, segundo seu poder tóxico. Essa classificação, fundamental para o conhecimento da toxicidade de determinado produto sob o ponto de vista de seus efeitos agudos, foi estabelecida pela Lei 7.082/89 (BRASIL, 1989) e abrange: Classe I extremamente tóxicos, Classe II - altamente tóxicos, Classe III - mediamente tóxicos e Classe IV - pouco ou muito pouco tóxicos.

A Agência Nacional de Vigilância Sanitária (ANVISA), órgão vinculado ao Ministério da Saúde, coordena o Sistema Nacional de Vigilância Toxicológica mediante regulamentação, análise, controle e fiscalização de produtos e serviços que envolvam riscos à saúde (agrotóxicos, componentes e afins e outras substâncias químicas de interesse toxicológico). A ANVISA também realiza a avaliação toxicológica para fins de registro dos agrotóxicos e a reavaliação de produtos já registrados, bem como normatiza e elabora regulamentos técnicos dos ingredientes ativos dos agrotóxicos.

Todos os riscos inerentes ao uso de agrotóxicos podem ser substancialmente reduzidos. Murray e Taylor (2000) propuseram diversas ações mitigatórias para lidar com os envenenamentos 
em países em desenvolvimento, entre as quais estão: eliminação dos compostos mais tóxicos, promoção do uso de equipamentos de proteção pessoal para aplicação dos produtos e redução do uso de agrotóxicos pelo melhoramento das técnicas e maquinário de aplicação. Outra ação que pode ser tomada para mitigar os danos causados pelos agrotóxicos envolve sua substituição por outros menos tóxicos e/ou menos impactantes ao meio ambiente, mas que ofereçam a mesma eficiência.

O objetivo deste trabalho consistiu em efetuar levantamento dos ingredientes ativos registrados no Brasil, bem como dos seus respectivos produtos formulados, e propor alternativas para substituição daqueles com maior toxicidade/impacto ambiental por outros que apresentem menor toxicidade/impacto ambiental nas principais culturas brasileiras.

\section{MATERIAL E MÉTODOS}

Realizou-se o levantamento de todos os ingredientes ativos de agrotóxicos e seus respectivos produtos formulados, registrados no Brasil, utilizando as bases de dados do Sistema de Informações sobre Agrotóxicos (SIA), criado pelo decreto nº 4.074 de 2002 (BRASIL, 2002) e do Sistema de Agrotóxicos Fitossanitários (AGROFIT). Essas bases de dados disponibilizam online as informações sobre os agrotóxicos registrados e autorizados para serem comercializados no Brasil.

O processo de indicação de substitutos menos tóxicos foi realizado separadamente para as culturas de arroz, cana-de-açúcar, café, citrus, milho, feijão, soja e pastagens. Inicialmente, para cada cultura, todos os Ingrediente Ativos (IA) foram ranqueados de acordo com a classificação toxicológica e ambiental de seus respectivos produtos formulados. Os produtos enquadrados na classe I em ambas as classificações (extremamente tóxicos e altamente perigosos ao meio ambiente) foram selecionados. Em seguida, por meio do desenvolvimento de um algoritmo em planilha eletrônica, foram averiguadas todas as possibilidades de substituição desses IA.

Os critérios estabelecidos para a seleção de substitutos incluíram, além da menor toxidade, a obrigatoriedade de serem do mesmo tipo (herbicida, fungicida, inseticida e outros) e apresentarem a mesma forma de aplicação (foliar, solo, no momento da pós emergência, etc.) dos IA a serem substituídos. A razão para tal similaridade baseou-se na premissa que produtos do mesmo tipo e aplicação similar podem responder a pragas semelhantes.

Uma vez definidos os $I A$, todos os seus produtos formulados que apresentassem classificação ambiental II, III ou IV foram considerados substitutos.

\section{RESULTADOS E DISCUSSÃO}

\section{1 CLASSIFICAÇÃO DOS AGROTÓXICOS BRASILEIROS}

Até a realização do presente estudo (2010) estavam registrados na ANVISA 880 produtos formulados a partir de 508 ingredientes ativos, pertencentes a 140 grupos químicos distintos. Desses, os mais numerosos são os organofosforados (70), seguidos pelos carbamatos (43), piretroides (42), inorgânicos (22), sulfonilireicos (19), triazóis (17), ácidos arilóxifenopropiônicos (15) e precursores de isocianato de metila (13).

Os IA foram distribuídos (Tabela 1) de acordo com a classificação toxicológica estipulada pela legislação brasileira em vigor. Observou-se que cerca de $19 \%$ de todos os IA pertencem à Classe Toxicológica I. O percentual dos produtos formulados com a mesma classificação toxicológica alcançou aproximadamente $21 \%$. Os produtos que são, simultaneamente, extremamente tóxicos e altamente perigosos ao ambiente representaram 3,9\% do total (34), conforme a Tabela 2. 
TABELA 1 - DISTRIBUIÇÃO DOS INGREDIENTES ATIVOS (IA) DAS DIFERENTES CLASSES DE AGROTÓXICOS DE ACORDO COM SEU NÍVEL DE TOXICIDADE

\begin{tabular}{ccccccc}
\hline Classe & I & II & III & IV & ND $^{*}$ & Total \\
\hline Acaricidas & 16 & 23 & 16 & 7 & 1 & $\mathbf{6 3}$ \\
\hline Bactericidas & 1 & 2 & 1 & 4 & 0 & $\mathbf{8}$ \\
\hline Cupinicidas & 5 & 2 & 0 & 0 & 0 & $\mathbf{7}$ \\
\hline Formicidas & 7 & 7 & 2 & 1 & 1 & $\mathbf{1 8}$ \\
\hline Fungicidas & 15 & 22 & 50 & 22 & 5 & $\mathbf{1 1 4}$ \\
\hline Herbicidas & 18 & 14 & 74 & 10 & 26 & $\mathbf{1 4 2}$ \\
\hline Inseticidas & 25 & 46 & 47 & 18 & 5 & $\mathbf{1 4 1}$ \\
\hline Nematicidas & 9 & 4 & 1 & 0 & 1 & $\mathbf{1 5}$ \\
\hline Total & $\mathbf{9 6}$ & $\mathbf{1 2 0}$ & $\mathbf{1 9 1}$ & $\mathbf{6 2}$ & $\mathbf{3 9}$ & $\mathbf{5 0 8}$ \\
\hline
\end{tabular}

* Classificação toxicológica não disponível.

\section{TABELA 2 - DISTRIBUIÇÃO DOS AGROTÓXICOS REGISTRADOS NO BRASIL DE ACORDO COM A CLASSIFICAÇÃO DE RISCO AMBIENTAL E TOXICIDADE}

\begin{tabular}{|c|c|c|c|c|c|}
\hline \multirow{2}{*}{$\begin{array}{l}\text { Classificação de } \\
\text { Risco Ambiental }\end{array}$} & \multicolumn{4}{|c|}{ Classificação Toxicológica } & \multirow{2}{*}{ Tota } \\
\hline & $\mathbf{I}$ & II & III & IV & \\
\hline I & 34 & 26 & 13 & 5 & 78 \\
\hline II & 115 & 130 & 156 & 52 & 453 \\
\hline III & 30 & 27 & 97 & 107 & 261 \\
\hline IV & 1 & 2 & 2 & 27 & 32 \\
\hline * & 7 & 21 & 21 & 7 & 56 \\
\hline Total & 187 & 206 & 289 & 198 & 880 \\
\hline
\end{tabular}

* Classificação de risco ambiental não disponível.

\subsection{ALTERNATIVAS PARA IA EXTREMAMENTE TÓXICOS E ALTAMENTE PERIGOSOS AO MEIO AMBIENTE}

Entre os agrotóxicos analisados, 34 apresentam simultaneamente as características de extremamente tóxicos e altamente perigosos ao meio ambiente. Os ingredientes ativos desses produtos são: 2,4-D, acetocloro, alacloro + trifluralina, azociclotina, brometo de metila, cipermetrina, ciproconazol, clorotalonil, clorpirifós, deltametrina + triazofós, dicofol, dinocape, endossulfan fenamifós, fosfeto de alumínio, fosfeto de magnésio, hidróxido de fentina, piridabem e terbufós. Seis desses ingredientes ativos são utilizados nas culturas estudadas (Tabela 3): 2,4-D, azociclotina, carbofurano, endossulfan, fenamifós e terbufós. Para pastagens não foi identificado nenhum produto formulado que apresentasse, simultaneamente, classificação toxicológica e ambiental nível I.

Ressalta-se que foge do escopo deste trabalho apresentar detalhamento da eficácia dos substitutos propostos para as espécies-pragas de cada uma das culturas analisadas. Cabe aos profissionais competentes, tais como químicos e engenheiros agrônomos, a tarefa de testar e validar a eficácia dos substituintes. 
TABELA 3 - RELAÇÃO DOS INGREDIENTES ATIVOS (IA) EXTREMAMENTE TÓXICOS E

ALTAMENTE PERIGOSOS AO MEIO AMBIENTE UTILIZADOS NAS CULTURAS

DE ARROZ, CANA-DE-AÇÚCAR, CAFÉ, CITRUS, MILHO, FEIJÃO E SOJA

\begin{tabular}{|c|c|c|c|c|c|c|c|}
\hline \multirow{2}{*}{ IA } & \multirow{2}{*}{ Cultura } & \multicolumn{6}{|c|}{ Tipos de agrotóxicos } \\
\hline & & acaricida & cupinicida & formicida & herbicida & inseticida & nematicida \\
\hline \multirow{5}{*}{$2,4-D$} & Arroz & & & & $x$ & & \\
\hline & Café & & & & $x$ & & \\
\hline & Cana & & & & $x$ & & \\
\hline & Milho & & & & $x$ & & \\
\hline & Soja & & & & $x$ & & \\
\hline \multirow{2}{*}{ azociclotina } & Feijão & $x$ & & & & & \\
\hline & Citrus & $x$ & & & & & \\
\hline \multirow{3}{*}{ carbofurano } & Arroz & $x$ & $x$ & & & $x$ & \\
\hline & Feijão & $x$ & $x$ & & & $x$ & \\
\hline & Milho & $x$ & $x$ & & & $x$ & \\
\hline \multirow{3}{*}{ endossulfan } & Café & $x$ & & $x$ & & $\mathrm{X}$ & \\
\hline & Cana & $x$ & & $x$ & & $x$ & \\
\hline & Soja & $x$ & & $x$ & & $x$ & \\
\hline fenamifós & Café & & & & & & $x$ \\
\hline \multirow{3}{*}{ terbufós } & Café & & & & & $x$ & $x$ \\
\hline & Cana & & & & & $x$ & \\
\hline & Milho & & & & & $x$ & \\
\hline
\end{tabular}

3.2.1 Substitutos para o 2,4-D

O ácido 2,4 diclorofenoxiacético (2,4-D) foi largamente utilizado na agricultura nos últimos cinquenta anos, especialmente em países em desenvolvimento. Entretanto, seu uso intensivo trouxe sérios problemas de contaminação do solo e do lençol freático (BOIVIN et al., 2005). Estudos apontam a relação entre o 2,4-D e distúrbios endócrinos em animais e seres humanos (FU et al., 2009)

Os 38 substitutos encontrados para o 2,4-D estão dispostos na Tabela 4, sendo que ametrina + simazina, azafenidina, metribuzim e simazina apresentam nível III de toxidade ambiental e podem ser utilizados nas culturas de arroz, café, cana, milho e soja.

\subsubsection{Substitutos para a Azociclotina}

A azociclotina, composto organoestânico, é bastante eficaz contra ácaros fitófagos e os impactos ambientais dos efeitos desse agrotóxico foram estudados em ambiente de água doce (FLIEDNER et al., 1997). Os resultados obtidos mostraram que sob concentrações nominais de $135 \mu \mathrm{g} \mathrm{L}^{-1}$, a azociclotina provocou severos efeitos na comunidade plantônica, incluindo fito e picoplânctons. Para os zooplânctons, os efeitos foram observados sob concentrações nominais de $45 \mu \mathrm{g} \mathrm{L}^{-1}$. Nos sedimentos, o aumento na atividade de degradação microbiana anaeróbia provocou alta mortalidade de plânctons. 
TABELA 4 - INGREDIENTES ATIVOS (IA) SUBSTITUTOS DOS PRODUTOS FORMULADOS A PARTIR DO 2,4-D PARA TODAS AS CULTURAS, QUE APRESENTAM APENAS AÇÃO HERBICIDA

\begin{tabular}{|c|c|c|c|c|c|}
\hline \multirow{2}{*}{ IA substituto } & \multicolumn{5}{|c|}{ Cultura } \\
\hline & Arroz & Café & Cana & Milho & Soja \\
\hline ametrina & & $\mathrm{x}$ & $x$ & $\mathrm{x}$ & \\
\hline ametrina + clomazona & & $x$ & & $x$ & \\
\hline ametrina + diurom & & $x$ & & $x$ & \\
\hline ametrina + diurom + MCPA & & $x$ & & $x$ & \\
\hline ametrina + simazina & & $x$ & & $x$ & \\
\hline ametrina + trifloxissulfurom-sódico & & $X$ & & $x$ & \\
\hline amicarbazona & & & $\mathrm{X}$ & & \\
\hline atrazina & & & $x$ & $x$ & \\
\hline atrazina + bentazona & & & & $\mathrm{X}$ & \\
\hline atrazina + dimetenamida & & & & $x$ & \\
\hline atrazina + isoxaflutol & & & & $x$ & \\
\hline atrazina + nicossulfurom & & & & $\mathrm{X}$ & \\
\hline atrazina + simazina & & & & $x$ & \\
\hline atrazina + S-metolacloro & & & & $X$ & \\
\hline atrazina+simazina & & & & $\mathrm{X}$ & \\
\hline azafenidina & & $\mathrm{x}$ & $\mathrm{X}$ & & \\
\hline azafenidina + hexazinona & & $x$ & $X$ & & \\
\hline diurom & & $\mathrm{X}$ & $\mathrm{X}$ & & \\
\hline diurom + dicloreto de paraquate & & $\mathrm{x}$ & $\mathrm{X}$ & & \\
\hline diurom + glifosato & & $\mathrm{x}$ & $\mathrm{x}$ & & \\
\hline diurom + hexazinona & & $X$ & $\mathrm{x}$ & & \\
\hline diurom + MSMA & & $\mathrm{X}$ & $\mathrm{X}$ & & \\
\hline diurom + tebutiurom & & $\mathrm{X}$ & $\mathrm{X}$ & & \\
\hline diurom + tidiazurom & & $\mathrm{X}$ & $\mathrm{X}$ & & \\
\hline etoxissulfurom & $x$ & & $X$ & & \\
\hline flazassulfurom & & & $x$ & & \\
\hline flumioxazina & & & & & $\mathrm{X}$ \\
\hline hexazinona & & & $\mathrm{X}$ & & \\
\hline imazapique & $\mathrm{X}$ & & $\mathrm{X}$ & & \\
\hline imazapique + imazapir & $\mathrm{x}$ & & $x$ & & \\
\hline imazapir & $\mathrm{x}$ & & & & \\
\hline imazaquim & & & & & $\mathrm{X}$ \\
\hline isoxaflutol & & & $\mathrm{X}$ & & \\
\hline linurom & & & & $x$ & $x$ \\
\hline metribuzim & & $x$ & $x$ & & \\
\hline oxadiazona & $\mathrm{X}$ & & & & \\
\hline pirazossulfurom-etílico & $\mathrm{X}$ & & & & \\
\hline simazina & & $x$ & $x$ & $X$ & \\
\hline Total & 6 & 17 & 20 & 16 & 3 \\
\hline
\end{tabular}

Os possíveis substitutos encontrados para a azociclotina estão dispostos na Tabela $5 \mathrm{e}$ podem ser utilizados nas culturas de citrus e feijão como acaricidas. 
TABELA 5 - INGREDIENTES ATIVOS (IA) SUBSTITUTOS DOS PRODUTOS FORMULADOS A PARTIR DA AZOCICLOTINA COM AÇÃO ACARICIDA EM TODAS AS CULTURAS

\begin{tabular}{ccc}
\hline IA substituto & Citrus & Feijão \\
\hline acefato & $\mathrm{X}$ & $\mathrm{X}$ \\
\hline amitraz & $\mathrm{X}$ & \\
\hline bifentrina & $\mathrm{X}$ & $\mathrm{X}$ \\
\hline bromopropilato & $\mathrm{X}$ & $\mathrm{X}$ \\
\hline carbossulfano & $\mathrm{X}$ & \\
\hline clofentezina & $\mathrm{X}$ & $\mathrm{X}$ \\
\hline clorfenapir & $\mathrm{X}$ & $\mathrm{X}$ \\
\hline clorpirifós & $\mathrm{X}$ & \\
\hline dicofol & $\mathrm{X}$ & \\
\hline diflubenzurom & $\mathrm{X}$ & \\
\hline dimetoato & $\mathrm{X}$ & $\mathrm{X}$ \\
\hline enxofre & $\mathrm{X}$ & $\mathrm{X}$ \\
\hline fenpropatrina & & $\mathrm{X}$ \\
\hline malationa & $\mathrm{X}$ \\
\hline mancozebe & $\mathrm{X}$ \\
\hline piridafentiona & $\mathrm{X}$ \\
\hline pirimifós-metil & $\mathrm{X}$ \\
\hline quinometionato & $\mathrm{X}$ \\
\hline tetradifona & & $\mathrm{X}$ \\
\hline triazofós & & \\
\hline Total geral & & $\mathbf{1 2}$ \\
\hline
\end{tabular}

\subsubsection{Substitutos para o Endossulfan}

O endossulfan, organoclorado com distribuição mundial, tem sido usado há cinco décadas por sua grande eficácia contra vários insetos e carrapatos transmissores de doenças (ROBERTS et al., 2003). Como resultado, esse agrotóxico foi aplicado em grande gama de plantações. Entretanto, devido à sua semi-volatilidade e persistência relativa, o produto representa potencial contaminante ambiental. A concentração de endossulfan no ar, no solo, na água e na vegetação tem sido reportada em diversos ambientes próximos a locais de aplicação (GARBARINO, SNYDER-CONN e LEIKER, 2002; CARRERA et al., 2002; POZO et al., 2006). Trata-se de um dos inseticidas mais comuns em corpos aquáticos nos Estados Unidos da América (SIDDIQUE, OKEKE e ARSHAD, 2003) e um dos organoclorados mais abundantes na atmosfera (SHEN et al., 2005).

O endossulfan foi considerado Poluente Orgânico Persistente (POP) por ter sido constatado em regiões distantes de seus locais de aplicação, tendo sido encontrado até na região ártica, tanto no ar, como na neve, no gelo, sedimentos e solo (FAN, 2008; USEPA, 2002a).

Organizações não governamentais têm efetuado diversas campanhas para a abolição total do endossulfan, devido ao seu potencial efeito carcinogênico e teratogênico. Esse agrotóxico foi pauta de polêmica no Brasil, pois a Agência Nacional de Vigilância Sanitária providenciou o banimento desse ingrediente ativo do mercado nacional a partir de julho de 2013 (ANVISA, 2010).

Os possíveis substitutos encontrados para o endossulfan estão dispostos nas Tabelas 6, 7 e 8 , 
respectivamente para as culturas de café, soja e cana. Dentre os substitutos, dois (enxofre e óleo mineral) enquadram-se no nível de classificação ambiental IV e 21 (expostos na Tabela 9) no nível III.

TABELA 6 - INGREDIENTES ATIVOS (IA) SUBSTITUTOS DOS PRODUTOS FORMULADOS A PARTIR DO ENDOSSULFAN PARA APLICAÇÃO NA CULTURA DE CAFÉ

\begin{tabular}{|c|c|c|c|}
\hline IA substituto & acaricidas & formicidas & inseticidas \\
\hline alfa-cipermetrina & & & $x$ \\
\hline beta-ciflutrina & & & $x$ \\
\hline ciflutrina & & & $x$ \\
\hline ciflutrina + imidacloprido & & & $x$ \\
\hline cipermetrina & & $x$ & $x$ \\
\hline cloridrato de cartape & & & $x$ \\
\hline clorpirifós & $x$ & $x$ & $x$ \\
\hline deltametrina & & $x$ & $x$ \\
\hline enxofre & $x$ & & \\
\hline esfenvalerato & & & $x$ \\
\hline esfenvalerato + fenitrotiona & & & $x$ \\
\hline espinosade & & & $x$ \\
\hline espirodiclofeno & $x$ & & \\
\hline etiona & $x$ & & $x$ \\
\hline fenpropatrina & $x$ & & $x$ \\
\hline fentiona & $x$ & & $x$ \\
\hline fluvalinato & $x$ & & $x$ \\
\hline hexitiazoxi & $x$ & & \\
\hline lambda-cialotrina & & & $x$ \\
\hline lufenurom & & & $x$ \\
\hline lufenurom + profenofós & & & $x$ \\
\hline malationa & $x$ & & $x$ \\
\hline mancozebe & $x$ & & \\
\hline óleo mineral & $x$ & & $x$ \\
\hline permetrina & & $x$ & $x$ \\
\hline piridafentiona & $x$ & & $x$ \\
\hline piriproxifem & & & $x$ \\
\hline profenofós & & & $x$ \\
\hline teflubenzurom & & & $x$ \\
\hline triazofós & $x$ & & $x$ \\
\hline zeta-cipermetrina & & & $x$ \\
\hline
\end{tabular}


TABELA 7 - INGREDIENTES ATIVOS (IA) SUBSTITUTOS DOS PRODUTOS FORMULADOS A PARTIR DO ENDOSSULFAN

PARA APLICAÇÃO NA CULTURA DE SOJA

\begin{tabular}{|c|c|c|c|}
\hline IA substituto & acaricidas & formicidas & inseticidas \\
\hline acefato & $x$ & & $x$ \\
\hline alfa-cipermetrina & & & $x$ \\
\hline beta-ciflutrina & & & $x$ \\
\hline beta-cipermetrina & & & $x$ \\
\hline bifentrina & $x$ & $x$ & $x$ \\
\hline ciflutrina & & & $x$ \\
\hline ciflutrina + imidacloprido & & & $x$ \\
\hline cipermetrina & & $\mathrm{x}$ & $\mathrm{x}$ \\
\hline cipermetrina + profenofós & & & $x$ \\
\hline clorfluazurom & & & $x$ \\
\hline clorpirifós & $x$ & $\mathrm{x}$ & $x$ \\
\hline deltametrina & & $x$ & $x$ \\
\hline diflubenzurom & $x$ & & $x$ \\
\hline enxofre & $x$ & & \\
\hline esfenvalerato & & & $x$ \\
\hline espinosade & & & $x$ \\
\hline etofenproxi & & & $x$ \\
\hline fenitrotiona & & $x$ & $x$ \\
\hline fenpropatrina & $x$ & & $\mathrm{x}$ \\
\hline fipronil & & $x$ & $x$ \\
\hline flufenoxurom & $x$ & & $x$ \\
\hline imidacloprido & & & $x$ \\
\hline lambda-cialotrina & & & $x$ \\
\hline lufenurom & & & $x$ \\
\hline lufenurom + profenofós & & & $x$ \\
\hline metoxifenozida & & & $x$ \\
\hline novalurom & & & $x$ \\
\hline permetrina & & $x$ & $x$ \\
\hline piriproxifem & & & $x$ \\
\hline profenofós & & & $x$ \\
\hline tebufenozida & & & $x$ \\
\hline teflubenzurom & & & $x$ \\
\hline tiacloprido & & & $x$ \\
\hline tiametoxam & & & $x$ \\
\hline tiodicarbe & & & $x$ \\
\hline triazofós & $x$ & & $x$ \\
\hline triclorfom & & & $x$ \\
\hline triflumurom & & & $x$ \\
\hline zeta-cipermetrina & & & $\mathrm{x}$ \\
\hline
\end{tabular}


TABELA 8 - INGREDIENTES ATIVOS (IA) SUBSTITUTOS DOS PRODUTOS FORMULADOS A PARTIR DO ENDOSSULFAN PARA APLICAÇÃO NA CULTURA DE CANA

\begin{tabular}{cccc}
\hline IA substituto & acaricidas & formicidas & inseticidas \\
\hline bifentrina & $\mathrm{X}$ & $\mathrm{X}$ & \\
\hline fipronil & & $\mathrm{X}$ & $\mathrm{X}$ \\
\hline imidacloprido & $\mathrm{X}$ & $\mathrm{X}$ & $\mathrm{X}$ \\
\hline tiametoxam & & & $\mathrm{X}$ \\
\hline triclorfom & & & $\mathrm{X}$ \\
\hline triflumurom & $\mathrm{X}$ & & $\mathrm{X}$ \\
\hline
\end{tabular}

TABELA 9 - INGREDIENTES ATIVOS (IA) SUBSTITUTOS DOS PRODUTOS FORMULADOS A PARTIR DO ENDOSSULFAN QUE APRESENTAM NÍVEL III DE CLASSIFICAÇÃO AMBIENTAL

\begin{tabular}{|c|c|c|}
\hline \multirow{2}{*}{ IA substituto } & \multicolumn{2}{|c|}{ Classificação } \\
\hline & Ambiental & Toxicológica \\
\hline acefato & III & III e IV \\
\hline bifentrina & III & III \\
\hline cipermetrina & III & II \\
\hline deltametrina & III & IV \\
\hline diflubenzurom & III & III e IV \\
\hline enxofre & III & IV \\
\hline espinosade & III & III \\
\hline espirodiclofeno & III & III \\
\hline etofenproxi & III & IV \\
\hline hexitiazoxi & III & III \\
\hline imidacloprido & III & III e IV \\
\hline malationa & III & II e III e IV \\
\hline mancozebe & III & III \\
\hline metoxifenozida & III & IV \\
\hline óleo mineral & III & IV \\
\hline tebufenozida & III & IV \\
\hline tiacloprido & III & III \\
\hline tiametoxam & III & III \\
\hline tiodicarbe & III & II e III \\
\hline triclorfom & III & II \\
\hline triflumurom & III & II e IV \\
\hline
\end{tabular}

\subsubsection{Substitutos para o Carbofurano}

O carbofurano, inseticida/nematicida de contato e sistêmico de amplo espectro, representa riscos ambientais por ser solúvel em água e apresentar grande mobilidade no solo, evidenciando elevado potencial para contaminação de lençóis freáticos.

Desde 1981 sabe-se que a aplicação de carbofurano causa degradação química do solo, a qual pode gerar perda na eficácia do produto (TRABUE et al., 2001). Por tais motivos, Plangklang e Reungsang (2010) defenderam a necessidade de banimento do carbofurano.

Os possíveis substitutos encontrados para o carbofurano estão dispostos na Tabela 10, 
sendo que acefato, benfuracarbe, bifentrina, imidacloprido, tiametoxam e tiodicarbe apresentam classificação ambiental nível III. Apenas o clorpirifós, utilizado como inseticida em plantações de milho, enquadra-se no nível ambiental IV.

\section{TABELA 10 - INGREDIENTES ATIVOS (IA) SUBSTITUTOS DOS PRODUTOS FORMULADOS A PARTIR DO CARBOFURANO}

\begin{tabular}{|c|c|c|c|c|}
\hline Cultura & IA substituto & acaricidas & cupinicidas & inseticidas \\
\hline \multirow{7}{*}{ Arroz } & benfuracarbe & & & $x$ \\
\hline & carbossulfano & $X$ & & $x$ \\
\hline & fipronil & & $x$ & $x$ \\
\hline & furatiocarbe & & & $x$ \\
\hline & imidacloprido & & & $x$ \\
\hline & tiametoxam & & & $x$ \\
\hline & tiodicarbe & & & $x$ \\
\hline \multirow{6}{*}{ Feijão } & acefato & $x$ & & $x$ \\
\hline & carbossulfano & $x$ & & $x$ \\
\hline & fipronil & & $X$ & $\mathrm{x}$ \\
\hline & furatiocarbe & & & $x$ \\
\hline & imidacloprido & & & $x$ \\
\hline & tiametoxam & & & $x$ \\
\hline \multirow{9}{*}{ Milho } & benfuracarbe & & & $x$ \\
\hline & bifentrina & $x$ & & $x$ \\
\hline & carbossulfano & $x$ & & $\mathrm{X}$ \\
\hline & clorpirifós & $X$ & & $x$ \\
\hline & fipronil & & $x$ & $x$ \\
\hline & furatiocarbe & & & $x$ \\
\hline & imidacloprido & & & $x$ \\
\hline & tiametoxam & & & $\mathrm{x}$ \\
\hline & tiodicarbe & & & $\mathrm{X}$ \\
\hline
\end{tabular}

\subsubsection{Substitutos para o Fenamifós}

O organoclorado fenamifós e seus produtos de degradação apresentam grande mobilidade e, portanto, alto potencial para alcançar lençóis freáticos e contaminar águas superficiais. Adicionalmente, evidências laboratoriais indicam que seus produtos de degradação são ainda mais móveis, potencializando o risco de contaminação (EPA, 2002b). O produto é extremamente tóxico para mamíferos, aves e organismos aquáticos, sendo que pequenas quantidades lançadas nos corpos aquáticos podem matar esses últimos (EPA, 2002b)

De acordo com os critérios estabelecidos neste trabalho, o único IA substituinte para o fenamifós é o fostiazato para a cultura de café, atuando como nematicida.

\subsubsection{Substitutos para o Terbufós}

O terbufós pertence ao grupo dos ditiofosforados, sendo bastante utilizado como nematicida e inseticida. O produto pode apresentar efeitos sinérgicos com herbicidas (CHEN, PEHKONEN e LAU, 2004). Na cana-de-açúcar, por exemplo, o controle de plantas daninhas é efetuado com herbicidas. Muitas áreas recebem nematicidas ou inseticidas no sulco de plantio e herbicidas logo a 
seguir, o que em certas condições resulta no aumento dos sintomas de fitointoxicação do herbicida (DINARDO-MIRANDA, 2001).

No Brasil, os primeiros registros de ocorrência de fitointoxicação causada por herbicidas em áreas tratadas com nematicida ocorreram na década de 1980, com a descrição de severas injúrias nas folhas de canaviais tratados com carbofurano no sulco de plantio e tebutiuron, em pré-emergência das plantas daninhas, logo após o plantio (BLANCO et al., 1980 apud DINARDOMIRANDA, GIL e GONÇALVES, 2006).

Dinardo-Miranda (2001) verificou que a aplicação de alguns herbicidas em área cultivada com cana-de-açúcar resultou na redução de produtividade nas parcelas tratadas com carbofuran ou terbufós.

Os possíveis substitutos encontrados para o terbufós estão dispostos na Tabela 11. Apenas o clorpirifós, utilizado como inseticida em plantações de milho, apresenta nível ambiental IV. Os IA substitutos que se enquadram na classificação ambiental III são a bifentrina, imidacloprido e tiametoxam.

\section{TABELA 11 - INGREDIENTES ATIVOS (IA) SUBSTITUTOS DOS PRODUTOS FORMULADOS A PARTIR DO TERBUFÓS}

\begin{tabular}{cccc}
\hline Cultura & substituto & inseticidas & nematicidas \\
\hline \multirow{3}{*}{ Café } & fostiazato & $X$ & $X$ \\
\cline { 2 - 3 } & imidacloprido & $X$ & \\
\cline { 2 - 3 } & tiametoxam & $X$ \\
\cline { 2 - 3 } Cana & bifentrina & $X$ \\
\cline { 2 - 3 } & fipronil & $X$ \\
\hline \multirow{2}{*}{ Milho } & imidacloprido & $X$ \\
& tiametoxam & $X$ \\
\hline & bifentrina & $X$ \\
\hline & clorpirifós & $X$ \\
\hline
\end{tabular}

\section{CONCLUSÃO}

No presente trabalho foram apontadas algumas alternativas menos tóxicas de agrotóxicos comercializados no mercado brasileiro, com o objetivo de reduzir seus impactos à saúde humana $e$ principalmente ao meio ambiente.

Os ingredientes ativos de classe I (altamente perigosos) 2,4-D, azociclotina, carbofurano, endossulfan, fenamifós e terbufós podem ser substituídos por diversos outros ingredientes ativos com periculosidade ambiental mais baixas.

Embora não tenham sido realizados testes mais detalhados sobre a eficiência das alternativas menos tóxicas frente as principais espécies-pragas das cultura analisadas, o estudo ressalta a importância da elaboração de programas de substituição de agrotóxicos com alta toxicidade, que avaliem os benefícios e os custos de tais substituições. Como no Brasil pesquisas dessa natureza são incipientes, embora já existam programas de racionalização do uso de agrotóxicos, acredita-se que este estudo possa servir como referência no sentido de auxiliar a formulação de políticas que reduzam os riscos ambientais inerentes ao uso desses insumos. 


\section{ABSTRACT \\ PROPOSAL FOR LESS TOXIC ALTERNATIVES TO PESTICIDES ACTIVE INGREDIENTS IN THE BRAZILIAN MARKET}

This study has identified the pesticide active ingredients (AI) registered in Brazil suggesting alternatives to substitute those with higher toxicity/environmental impact in cultures of rice, sugarcane, coffee, citrus, maize, bean, soybean and pastures. Substitution was proposed for 2,4-D, azocyclotin, carbofuran, endossulfan, fenamifos and terbufos. It is expected that this study may contribute as a reference on the improvement of agrienvironmental policy design able to reduce environmental risks inherent to the use of these substances.

KEY-WORDS: PESTICIDES; ALTERNATIVE ACTIVE INGREDIENTS; ENVIRONMENTAL RISKS.

\section{REFERÊNCIAS}

1 Agência Nacional de Vigilância Sanitária (ANVISA). RDC nº 28 de 9 de agosto de 2010. Aprova o regulamento técnico para o ingrediente ativo endossulfam em decorrência da reavaliação toxicológica. Diário Oficial [da] República Federativa do Brasil, Brasília, DF, n.156, 16 ago. 2010, Seção I, p. 64.

2 BOIVIN, A.; AMELLAL, S.; SCHIAVON, M.; VAN GENUCHTEN, M. 2,4-dichlorophenoxyacetic acid (2,4-D) sorption and degradation dynamics in three agricultural soils. Environmental Pollution, v.138, p.92-99, 2005.

3 BRASIL. Presidência da República. Decreto n. 4.074, de 04 de janeiro de 2002. Regulamenta a Lei no 7.802, de 11 de julho de 1989, que dispõe sobre a pesquisa, a experimentação, a produção, a embalagem e rotulagem, o transporte, o armazenamento, a comercialização, a propaganda comercial, a utilização, a importação, a exportação, o destino final dos resíduos e embalagens, o registro, a classificação, o controle, a inspeção e a fiscalização de agrotóxicos, seus componentes e afins. Diário Oficial [da] República Federativa do Brasil, Brasília, DF, 08 jan. 2002, Seção I, p.01.

4 BRASIL. Presidência da República. Lei n. 7.092, de 11 de julho de 1989. Dispõe sobre a pesquisa, a experimentação, a produção, a embalagem e rotulagem, o transporte, o armazenamento, a comercialização, a propaganda comercial, a utilização, a importação, a exportação, o destino final dos resíduos e embalagens, o registro, a classificação, o controle, a inspeção e a fiscalização de agrotóxicos, seus componentes e afins. Diário Oficial [da] República Federativa do Brasil, Brasília, DF, 14 jul. 1989, Seção III, p.56.

5 CARRERA, G.; FERNÁNDEZ, P.; GRIMALT, J.O.; VENTURA, M.; CAMARERO, L.; CATALAN, J.; NICKUS, U.; THIES, H.; PSENNER, R. Atmospheric deposition of organochlorine compounds to remote high mountain lakes of Europe. Environ. Sci. Technol., v.36, p.2581-2588, 2002.

6 CARSON, R. Silent spring. Boston: Houghton Mifflin, 1962. 378 p.

7 CHEN, J.; PEHKONEN, S.; LAU, C. Phorate and terbufos adsorption onto four tropical soils. Colloids and Surfaces A: Physicochemical and Engineering Aspects, v.240, n.1-3, p.55-61, 2004.

8 COSTA, C.; SILVA, S.; COELHO, P.; ROMA-TORRES, J.; TEIXEIRA, J. P.; MAYAN, O. Micronucleus analysis in a Portuguese population exposed to pesticides: preliminary survey. Int. J. Hyg. Environ. Health, v.210, p.415-418, 2007.

9 DABROWSKI, J. M.; PEALL, S. K. C.; REINECKE, A. J.; LIESS, M.; SCHULZ, R. Runoff-related pesticide input into the Lourens River, South Africa: basic data for exposure assessment and risk mitigation at the catchment scale. Water Air Soil Pollut., v.135, p.265-283, 2002.

10 DANIELS, J.L.; OLShAN, A.F.; SAVITZ, D.A. Pesticides and childhood cancers. Environ. Health Perspect., v.105, p.1068-1077, 1997.

11 DINARDO-MIRANDA, L. L. Efeitos da interação entre nematicidas e herbicidas em cana-de-açúcar. Nematologia Bras., v.25, n.2, p.197-204, 2001.

12 DINARDO-MIRANDA, L.L.; GIL, M.A.; GONCALVES, R.F. Interação entre nematicidas e herbicidas aplicados no plantio da cana-de-açúcar. Planta Daninha, v.24, n.3, p.557-562, 2006.

13 ENGEL, L.; CHECKOWAY, H.; KEIFER, M.; SEIXAS, N.; LONGSTRETH, W.; SCOTT, K.; HUDNELL, K.; ANGER, W. Parkinsonism and occupational exposure to pesticides. Occup. Environ. Med., v.58, p.581-589, 2001.

14 ERGENE, S.; CELIK, A.; CAVAŞ, T. Genotoxic biomonitoring study of population residing in pesticide contaminated regions in Göksu Delta: micronucleus, chromosomal aberrations and sister chromatid exchanges. Environ. Int., v.33, p. 877-885, 2007.

15 FAN, S. Endosulfan risk characterization document. Environmental fate. v.3. Environmental Monitoring Branch, Department of Pesticide Regulation, California Environmental Protection Agency, Sacramento, CA. 2008. Disponível em: <http://www.cdpr.ca.gov/docs/emon/pubs/tac/finaleva/endosulfan.htm>. Acesso em: 15 abr. 2010. 
16 FIGÁ-TALAMANCA, I.; PETRELLI G. Reduction in fertility in male greenhouse workers exposed to pesticides. Eur. J. Epidemiol., v.17, p.675-677, 2001.

17 FLIEDNER, A.; REMDE, A.; NIEMANN, R.; SCHÄFERS, C. Effects of the organotin pesticide azocyclotin in aquatic microcosms. Chemosphere, v.35, p.13-20, 1997.

18 FU, F.; XIAO, L.; WANG, W.; XU, X.; XU, L.; QI, G.; CHEN, G. Study on the degradation of 2,4-dichlorophenoxyacetic acid $(2,4-D)$ and 2-methyl-4-chloro-phenoxyacetic sodium (MCPA sodium) in natural agriculture-soils of Fuzhou, China using capillary electrophoresis. The Science of the Total Environment, v.407, n.6, p.1998-2003, 2008.

19 GARBARINO, J.; SNYDER-CONN, E.; LEIKER, T.J. Contaminants in Arctic snow collected over northwest Alaskan sea ice. Water Air Soil Pollut., v.139 p.183-214, 2002.

20 GARCIA, A. M. Occupational exposure to pesticides and congenital malformations: a review of mechanisms, methods, and results. Am. J. Ind. Med., v.33, p.232-240, 1998.

21 GAUTHIER E.; FORTIER I.; COURCHESNE F.; PEPIN P.; MORTIMER J.; GAUVREAU, D. Environmental pesticide exposure as a risk factor for Alzheimer's disease: a case-control study. Environ. Res., v.86, p.37-45, 2001.

22 Instituto Brasileiro do Meio Ambiente (IBAMA). Instrução Normativa n. 084 de 15 de outubro de 1996. Estabelece critérios a serem utilizados junto ao IBAMA, para efeito de registro e avaliação do potencial de periculosidade ambiental (PPA) de agrotóxicos, seus componentes e afins. Diário Oficial [da] República Federativa do Brasil, Brasília, DF, 15 out. 1996 , Seção I, p.124.

23 KHUDER, S.A.; MUTGI, A.B. Meta-analyses of multiple myeloma and farming. Am. J. Ind. Med., v.32, p.510-516, 1997.

24 MOURAD, T.A. Adverse impact of insecticides on the health of Palestinian farmworkers in the Gaza Strip: a hematologic biomarker study. Int. J. Occup. Environ. Health, v.11, p.144-149, 2005.

25 MUNIZ, J.F.; MCCAULEY, L.; SCHERER, J.; LASAREV, M.; KOSHY, M.; KOW, Y. W. Biomarkers of oxidative stress and DNA damage in agricultural workers: a pilot study. Toxicol. Appl. Pharmacol., v.227, p.97-107, 2008.

26 MURRAY, D.L.; TAYLOR, P.L. Claim no easy victories: evaluating the pesticide industry's global safe use campaign. World Dev., v.28, p.1735-1749, 2000.

27 OJAJARVI, I.; PARTANEN, T.; AHLBOM, A.; BOFFETT, P.; HAKULINEN, T.; JOURENKOVA, N. Occupational exposures and pancreatic cancer: a meta-analysis. Occup. Environ. Med., v.57, p.316-24, 2000.

28 PLANGKLANG, P.; REUNGSANG, A. Bioaugmentation of carbofuran by Burkholderia cepacia PCL3 in a bioslurry phase sequencing batch reactor. Process Biochemistry, v.45, n.2, p.230-238, 2010.

29 POZO, K.; HARNER, T.; WANIA, F.; MUIR, D. C. G.; JONES, K.C.; BARRIE, L. A. Toward a global network for persistent organic pollutants in air: results from the GAPS study. Environ. Sci. Technol., v.40, p.4867-4873, 2006.

30 RATTNER, B. A. History of wildlife toxicology. Ecotoxicology, v.18, n.7, p.286-291, 2009.

31 RISSATO, S. R.; LIBÂNIO, M.; GIAFFERIS, G. P.; GERENUTTI, M. Diagnóstico ambiental de metais e organoclorados em córregos adjacentes a áreas de cultivo de cana-de-açúcar (Estado de São Paulo, Brasil). Quim. Nova, v.27, p.739$748,2004$.

32 ROBERTS, D. M.; KARUNARATHNA, A.; BUCKLEY, N. A.; MANUWEERA, G.; SHERIFF, M. H. R.; EDDLESTON, M. Influence of pesticide regulation on acute poisoning deaths in Sri Lanka. Bull. World Health Organ., v.8, p.789-798, 2003.

33 SHEN, L.; WANIA, F.; LEI, Y. D.; TEIXEIRA, C.; MUIR, D. C.; BIDLEMAN, T. F. Atmospheric distribution and long-range transport behavior of organochlorine pesticides in North America. Environ. Sci. Technol., v.39, p.409-20, 2005.

34 SIDDIQUE, T.; OKEKE, B. C.; ARSHAD, M. Biodegradation kinetics of endosulfan by Fusarium ventricosum and a Pandoraea species. J. Agric. Food Chem., v.51, p.8015-8019, 2003.

35 USEPA. U.S. Environmental Protection Agency. Interim Reregistration Eligibility Decision (IRED) Fenamiphos. EPA 738-R-02-004. Washington, DC., 2002b. Disponível em: http://www.epa.gov/oppsrrd1/REDs/fenamiphos_ired.pdf Acesso em: 16 abr. 2010.

36 USEPA. U.S. Environmental Protection Agency. Reregistration Eligibility Decision for Endosulfan. Washington, DC., 2002a. Disponível em: http://www.epa.gov/oppsrrd 1/REDs/endosulfan_ired.pdf Acesso em: 15 abr. 2010.

37 ZAHM, S. H.; WARD, M. H. Pesticides and childhood cancer. Environ. Health Perpect., v.106, p.893-908, 1998. 